SECTION 1. Theoretical research in mathematics.

Nurmatov Yodgormirza Khatammirzayevich Head of the department of pediatric urology adolescent gynecology of the Namangan regional children's multi-disciplinary medical center. Tashkent Institute of Postgraduate Medical Education, Tashkent, Uzbekistan yodgormirzo@rambler.ru

Beknazarov Zhumanazar Beknazarovich $\mathrm{PhD}, \mathrm{ScD}$

Tashkent Institute of Postgraduate Medical Education, Tashkent, Uzbekistan

Kholmurodov Mamatkhon Kuchkarovich $\mathrm{PhD}, \mathrm{ScD}$, Namangan State University, Namangan, Uzbekistan

\title{
MATHEMATICAL MODELING AND FORECASTING COEFFICIENT URETHRAL RESISTANCE IN THE NEW SURGICAL TREATMENT OF URETHRAL VALVES IN CHILDREN.
}

Abstract: Mathematical modeling - a scientific approach that is associated with the construction and use of a mathematical model of the phenomenon under investigation, the subject or object, as well as systems, incorporating them to reduce the time, effort and money on a prediction of a possible future, improve the validity and accuracy of scientific predictions, regardless of their activity in. This paper presents the results of research and treatments in 71 children (from two months to 15 years of age) with urethral valves. In all, 28 patients were treated using the endoscopic method, and 43 patients treated by the proposed method, using a metallic urethrotome. Diagnosis of the urethral valves was done using ultrasonography, voiding cystourethrogram, urethrocystoscopy, uroflowmetry and cystomanomerty. Mathematical modeling was applied to assess the treatment methods. The proposed method, using the metallic urethrotome, was preferable to the endoscopic method.

Key words: mathematical modeling, urethral valves, coefficient urethral resistance, metallic urethrotome, endoscopic method.

УДК 616.62-007.2-053.2-07-089

\section{МАТЕМАТИЧЕСКОЕ МОДЕЛИРОВАНИЯ И ПРОГНОЗИРОВАНИЯ КОЭФФИЦИЕНТА УРЕТРАЛЬНОГО СОПРОТИВЛЕНИЯ НОВОМ ХИРУРГИЧЕСКОМ ЛЕЧЕНИИ ПРИ КЛАПАНАХ УРЕТРЫ У ДЕТЕЙ.}

Аннотация: Математическое моделирование - научный подход, связанный с построением и использованием математической модели исследуемого явления с иелью сокращения времени, сил и средств по предсказанию возможного будущего, повышения обоснованности и точности научных прогнозов, учёта их в деятельности. В статье изложены результаты исследований клапанов уретры у 71 детей, в возрасте от двух месячев до 15 лет. 28 из пациентов прошли лечение по эндоскопическому методу и 43 пациентов лечение по предложенной методике клиники (металлическим вальватомом). При диагностировании были применены методы УЗИ, микиион изистоуретрограмми, уретроцистоскопии, урофлоуметрии, иистоманометрии и их математические анализы. 


Ключевые слова: математическое моделирования, клапан уретры,
коэфффициент уретрального сопротивления, эндоскопический метод, металл
вальватом.

\section{Актуальность работы:}

Основой математического моделирования в медицине является имитация процесса обслуживания потока больных, что используется при планировании организационных мероприятий, в частности, для расчетов необходимого ресурсного обеспечения при задаваемом качестве медицинского обслуживания. Имитационное моделирование находит свое применение и при проектировании интегрированных информационных систем больниц[1,2].

На кафедре детской хирургии Ташкентского Института Усовершенствования врачей применяется программное изучения диагностики и прогнозирования эффективности лечения больных при помощи методов математического моделирования. На примере: Клапаны уретры (КУ) являются одними из тяжелых видов врождённой патологии, в частности, инфравезикальной обструкции, приводящих к нарушению уродинамики, развитию пиелонефрита и хронической болезни почек (ХБП), вопрос тактики лечения которой остаётся в ряду актуальных проблем хирургии детского возраста [3,4].

Наличие КУ у плода приводит к накоплению мочи в мочевом пузыре, вследствие чего повышается внутрипузырное давление, расширяется мочевой пузырь, появляется уретерогидронефроз, в результате чего развивается олигогидроамниоз, уринома, ренальная дисплазия, уринарный асцит и респираторные расстройства[5,6].

Вследствие происходящих изменений страдает функция почек, - от дисплазии до глубоких патологических изменений в паренхиме. Несмотря на адекватное лечение КУ, хроническая почечная недостаточность (ХПН) развивалась у 48-70\% больных $[7,8]$. Указанные патологические изменения не позволяют значительно улучшить результаты хирургического лечения, и летальность от ХПН остается относительно высокой, от 10,0 до 44\% и до настоящего времени остается в тех же пределах $[9,10]$.

Прогресс и усовершенствование эндоскопических инструментов позволяют разрушать клапаны коагуляционными электродами или миниатюрными ножами. Однако первичная трансуретральная резекция КУ у новорожденных травматична, иногда очень трудно избежать ятрогенного повреждения уретры из-за размера и узости наружного отверстия мочеиспускательного канала [11,12]. После трансуретральной резекции КУ у детей сужение уретры развивалось в $9 \%$ случаев, а у новорожденных - в $50 \%[13,14]$.

Несмотря на активную разработку новых принципов лечения и усовершенствования известных методов операции, результаты по-прежнему не удовлетворяли практикующих врачей. Частота рецидива после первичной аблации клапана достигает $20-45 \%$, иногда $[15,16]$, при этом в $12-56 \%$ случаев клапан сохранялся [17,18].

Все вышеуказанное обусловило к изучению результатов разрушения клапана уретры у детей, и разработки нового простого и малоинвазивного хирургического метода лечения клапана уретры у детей.

Для построения математической модели нами проанализированы клинические симптомы инфравезикальной обструкции, обусловленной клапанами уретры до и после оперативного лечения, на основании клинических симптомов и подтвержденной такими исследованиями, как ультразвуковая сонография, микционная цистоуретрография, урофлоуметрия, цистоманометрия, цистоскопия.

\section{Цель исследования}


Изучить применение математического моделирования при определение эффективности и прогнозировании коэффициента уретрального сопротивления при лечении клапана уретры у детей, с внедрением в практику нового метода, отличающийся простотой выполнения при минимальном хирургическом вмешательстве, отсутствием необходимости дорогостоящей аппаратуры, осуществляемые даже в лечебных учреждениях в странах и регионах со слаборазвитой экономикой.

\section{Материал и методы}

Изложены результаты проведенных исследований и лечебно-профилактических мероприятий начиная с 1998 по 2011 г.г. клапанов уретры у 71 детей, в возрасте от двух месяцев до 15 лет. При диагностировании были применены методы УЗИ, микцион цистоуретрограммы, уретроцистоскопии, урофлоуметрии, цистоманометрии и их математические анализы.

Клапаны уретры были ликвидированы у 28 больных путем эндоскопического удаления, а у 43 больных с помощью клапаноудаляющего инструмента, металлическим вальватомом, (патент на полезный модель UZ № FAP 20090046 23.08.2010) предложенного в клинике для удаления клапана уретры.

\section{Результаты и их обсуждение}

Статистическая обработка, разработка выявленных результатов и их цифровое графическое представление проводились на компьютере типа «Pentium-4» с использованием стандартных («Excel-2007»», « Statgrafics full», «Statistica 11.3») и специально разработанных программных средств, обеспечивающих эффективное применение методов математической модели и статистического анализа.

Применялисъ методы многомерного статистического анализа (позволяющий выделить наиболее значимых факторов риска и получить приемлемую модель для дальнейшего профилактического вмешательства), ранговый корреляционный анализ по методам Пирсона, Спирмена и Кэндалла U-критерий, критерий углового преобразования Фишера, критерий согласия Пирсона $\left(\mathrm{X}^{2}\right)$, критерий относительного риска ( $\mathrm{RR}$ uI R/R) и тест Шеллинга-Вольфейля ( для определения различий) [19,20].

Для оценки статистически достоверности рассчитанных критериев использовались показатели степени свободы (к) и таблицы критических значений для приемлемых уровней значимости (р). Для статистики различии были приняты четыре основных уровня достоверности : недостоверный (незначимый) - $p>0,050$, предельный (низкий) - $\mathrm{p}<$ 0,50 , средний $-\mathrm{p}<0,10$, высокий $-\mathrm{p}<0,001$. Основными верификаторами достоверности различия служили результаты универсальных (многофункциональных) методов Фишера и Шеллинга - Вольфейля. (Малета Ю.С.) [21,22].

Используя возможные разные виды уравнений множественной регрессии: линейные и нелинейные.

Ввиду четкой интерпретации параметров наиболее широко используется линейная функция. В линейной множественной регрессии

$$
y_{x}=a+b_{1} x_{1}+b_{2} x_{2}+\ldots+b_{m} x_{m}
$$

параметры при $x$ называются коэффициентами «чистой» регрессии. Они характеризуют среднее изменение результата с изменением соответствующего фактора на единицу при неизмененном значении других факторов, закрепленных на среднем уровне (1).

Рассмотрим линейную модель множественной регрессии

$$
y=a+b_{1} x_{1}+b_{2} x_{2}+\ldots+b_{m} x_{m}+\varepsilon .
$$

Классический подход к оцениванию параметров линейной модели множественной регрессии основан на методе наименьших квадратов (МНК). МНК позволяет получить 
такие оценки параметров, при которых сумма квадратов отклонений фактических значений результативного признака ${ }^{y}$ от расчетных ${ }^{y}$ минимальна (2):

$$
\sum_{i}\left(y_{i}-y_{x_{i}}\right)^{2} \rightarrow \min
$$

Как известно из курса математического анализа, для того чтобы найти экстремум функции нескольких переменных, надо вычислить частные производные первого порядка по каждому из параметров и приравнять их к нулю (3).

Итак, имеем функцию $m+1$ аргумента:

$$
S\left(a, b_{1}, b_{2}, \ldots, b_{m}\right)=\sum\left(y-a-b_{1} x_{1}-b_{2} x_{2}-\ldots-b_{m} x_{m}\right)^{2} .
$$

Находим частные производные первого порядка:

$$
\left\{\begin{array}{l}
\frac{\partial S}{\partial a}=-2 \sum\left(y-a-b_{1} x_{1}-b_{2} x_{2}-\ldots-b_{m} x_{m}\right)=0 \\
\frac{\partial S}{\partial b_{1}}=-2 \sum x_{1}\left(y-a-b_{1} x_{1}-b_{2} x_{2}-\ldots-b_{m} x_{m}\right)=0 \\
\ldots \ldots \ldots \ldots \ldots \ldots \ldots \ldots \ldots \ldots \ldots \ldots \ldots \ldots \ldots \ldots \ldots \ldots \ldots \ldots \ldots \\
\frac{\partial S}{\partial b_{m}}=-2 \sum x_{m}\left(y-a-b_{1} x_{1}-b_{2} x_{2}-\ldots . b_{m} x_{m}\right)=0
\end{array}\right.
$$

После элементарных преобразований приходим к системе линейных нормальных уравнений для нахождения параметров линейного уравнения множественной регрессии (4):

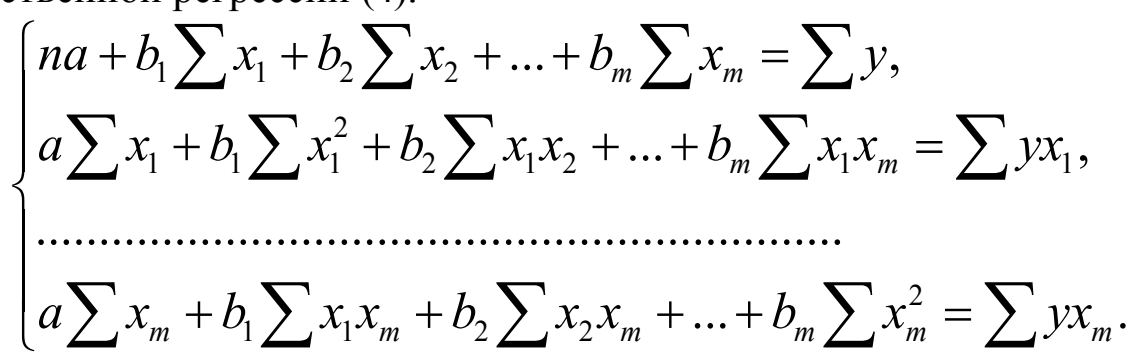

Метод наименьших квадратов применим и к уравнению множественной регрессии в стандартизированном масштабе (5)

$$
t_{y}=\beta_{1} t_{x_{1}}+\beta_{2} t_{x_{2}}+\ldots+\beta_{m} t_{x_{m}}+\varepsilon
$$

где $t_{y}, t_{x_{1}}, \ldots, t_{x_{m}}$ - стандартизированные переменные:

$t_{y}=\frac{y-\bar{y}}{\sigma_{y}}, t_{x_{i}}=\frac{x_{i}-\bar{x}_{i}}{\sigma_{x_{i}}}$, для которых среднее значение равно нулю: $\bar{t}_{y}=\bar{t}_{x_{i}}=0$, a среднее квадратическое отклонение равно единице: $\sigma_{t_{y}}=\sigma_{t_{x_{i}}}=1 ; \quad \beta_{i}$ стандартизированные коэффициенты регрессии.

Стандартизованные коэффициенты регрессии показывают, на сколько единиц изменится в среднем результат, если соответствующий фактор $x_{i}$ изменится на одну единицу при неизменном среднем уровне других факторов. В силу того, что все переменные заданы как центрированные и нормированные, стандартизованные 
коэффициенты регрессии $\beta_{i}$ можно сравнивать между собой. Сравнивая их друг с другом, можно ранжировать факторы по силе их воздействия на результат.

На следующей этапе статистический анализ модели и в первую очередь статистическое оценивание неизвестных параметров модели, а также сопоставление реальных и модельных данных, проверка адекватности модели, оценка точности модельных данных.

Оценка значимости уравнения регрессии в целом производится на основе $F$ критерия Фишера, которому предшествует дисперсионный анализ. В математической статистике дисперсионный анализ рассматривается как самостоятельный инструмент статистического анализа. В практике он применяется как вспомогательное средство для изучения качества регрессионной модели.

Определение дисперсии на одну степень свободы приводит дисперсии к сравнимому виду. Сопоставляя факторную и остаточную дисперсии в расчете на одну степень свободы, получим величину $F$-критерия Фишера (6):

$$
F=\frac{S_{\text {факт }}^{2}}{S_{\text {ост }}^{2}} .
$$

Фактическое значение $F$-критерия Фишера (9) сравнивается с табличным значением $F_{\text {табл }}\left(\alpha ; k_{1} ; k_{2}\right)$ при уровне значимости $\alpha$ и степенях свободы $k_{1}=m$ и $k_{2}=n-m-1$

( $n$ - число наблюдений, $m$ - число параметров при переменной $x$ ).

При этом, если фактическое значение $F$-критерия больше табличного, то признается статистическая значимость уравнения в целом. $F$-критерия Фишера (7) можно представить в виде

$$
F=\frac{S_{\text {факт }}^{2}}{S_{\text {ост }}^{2}}=\frac{\sum\left(\hat{y}_{x}-\bar{y}\right)^{2}}{\left(y-\hat{y}_{x}\right)^{2}} \cdot \frac{n-m-1}{m} .
$$

Используя методов наименьших квадратов, вычислены коэффициенты и получены математические модели.

Математическая модель коэффициента уретрального сопротивления определяется следующим образом (8).

$$
\mathbf{y}=\mathbf{a}_{0}+\mathbf{a}_{1} \mathbf{x}_{1}+\mathbf{a}_{2} \mathbf{x}_{2}+\mathbf{a}_{3} \mathbf{x}_{3}+\mathbf{a} 4 \mathbf{x}_{4}+\mathbf{a} 5 \mathbf{x}_{5}+\mathbf{a} \mathbf{x}_{6},
$$

где, у - коэффициент уретрального сопротивления;

$\mathrm{x}_{1}$ - максимальный объём мочевого пузыря;

$\mathrm{x}_{2}$ - толщина стенки мочевого пузыря;

$\mathrm{x}_{3}$ - объём остаточной мочи;

$\mathrm{x}_{4}$ - скорость мочеиспускания (сред);

Х5 - живое сечение уретры;

х6-- мышечное напряжение детрузора,

$\mathrm{a}_{0}, \mathrm{a}_{1}, \ldots, \mathrm{a}_{5}$ - постоянные числа.

Для определения коэффициентов $\mathrm{a}_{0}, \mathrm{a}_{1}, \ldots, \mathrm{a}_{5}$ используем из методов наименьших квадратов математической статистики.

1.Эндоскопическое иссечение клапана уретры:

- до операции:

$y=-3,6450 \cdot 10^{-2}-4,1601 \cdot 10^{-4} x_{1}+0,3024 x_{2}+3,0652 \cdot 10^{-3} x_{3}+8,3276 \cdot 10^{-3} x_{4}+0,1910$ $x_{5}+0,00235 \mathrm{x}_{6}$;

- после операции:

$y=8,8523 \cdot 10^{-3}-6,9645 \cdot 10^{-4} x_{1}+0,3110 x_{2}+9,1819 \cdot 10^{-3} x_{3}+0,1944 x_{4}+5,1634 x_{5}+0,00153$ $\mathrm{x}_{6}$; 
Табличное значение $F$-критерия при пятипроцентном уровне значимости ( $\left.\alpha=0,05, k_{1}=5, k_{2}=32-5-1=26\right): F_{\text {табл }}=2,62$. Так как $F_{\text {факт }}=25,76>F_{\text {табл }}=2,62$, то уравнение признается статистически значимым.

2. Удаление клапана уретры металлическим вальватомом:

- до операщии:

$y=-5,234 \cdot 10^{-3}+6,668 \cdot 10^{-4} x_{1}+0,355 x_{2}+3,922 \cdot 10^{-3} x_{3}-1,02 \cdot 10^{-3} x_{4}+0,253 x_{5}+0,00127 \mathrm{x}_{6} ;$

- после операиии:

$y=1,9144 \cdot 10^{-4}+1,3034 \cdot 10^{-4} x_{1}+4,7047 \cdot 10^{-3} x_{2}+7,0315 \cdot 10^{-3} x_{3}+3,4680 \cdot 10^{-4} x_{4}+$ $2,9779 \cdot 10^{-2} x_{5}+0,00234 \mathrm{x}_{6}$.

Табличное значение $F$-критерия при пятипроцентном уровне значимости ( $\left.\alpha=0,05, k_{1}=5, k_{2}=61-5-1=55\right): F_{\text {табл }}=2,38$. Так как $F_{\text {факт }}=49,87>F_{\text {табл }}=2,38$, то уравнение признается статистически значимым.

Достоверность коэффициентов математических моделей проверена с помощью статистики Фишера, и они являются значимыми с вероятностью $\mathrm{p}=0,95$. Используя методов наименьших квадратов, вычислены коэффициенты и получены математические модели. (табл.1).

Таблица 1

Показатели уродинамики мочеиспускания до и после операции удаления клапана уретры

\begin{tabular}{|c|c|c|c|c|}
\hline \multirow{2}{*}{$\begin{array}{c}\text { Показатели } \\
\text { исследования }\end{array}$} & \multicolumn{2}{|c|}{$\begin{array}{c}\text { Эндоскопическое удаление } \\
\text { КУ }\end{array}$} & \multicolumn{2}{|c|}{$\begin{array}{c}\text { Удаление КУ металлическим } \\
\text { вальватомом }\end{array}$} \\
\hline & до операции & $\begin{array}{c}\text { после } \\
\text { операции }\end{array}$ & до операции & $\begin{array}{c}\text { после } \\
\text { операции } \\
\end{array}$ \\
\hline $\begin{array}{lr}\text { Толщина } & \text { стенки } \\
\text { мочевого } \\
\text { (см) }\end{array}$ & $0,62 \pm 0,08^{*}$ & $0,54 \pm 0,02 *$ & $0,59 \pm 0,07^{*}$ & $0,50 \pm 0,05 * *$ \\
\hline $\begin{array}{l}\text { Максимальный } \\
\text { объем } \\
\text { пузыря }\end{array}$ & $162,7 \pm 37,2 *$ & $147,5 \pm 31,91 *$ & $190,2 \pm 34,99 *$ & $180,61 \pm 23,39 *$ \\
\hline $\begin{array}{l}\text { Объём ост. мочи } \\
\text { (мл) }\end{array}$ & $45,11 \pm 19,40 *$ & $11,19 \pm 7,19 *$ & $40,48 \pm 12,4 *$ & $8,93 \pm 2,28 * *$ \\
\hline $\begin{array}{l}\text { Живое сечение } \\
\text { уретры }\end{array}$ & $0,30 \pm 0,04^{*}$ & $0,47 \pm 0,04^{*}$ & $0,31 \pm 0,03 *$ & $0,50 \pm 0,03 * *$ \\
\hline $\begin{array}{l}\text { Коэффициент } \\
\text { уретрального } \\
\text { сопротивления }\end{array}$ & $0,28 \pm 0,07 *$ & $0,11 \pm 0,06^{*}$ & $0,22 \pm 0,06^{*}$ & $0,05 \pm 0,02 * *$ \\
\hline $\begin{array}{l}\text { Скорость } \\
\text { мочеиспускания } \\
\text { (мл/с) }\end{array}$ & $6,98 \pm 0,88^{*}$ & $13,42 \pm 0,81 * *$ & $7,52 \pm 0,74^{*}$ & $16,36 \pm 0,60 * *$ \\
\hline $\begin{array}{l}\text { Внутрипузырное } \\
\text { давление (мм.вод. } \\
\text { ст.) }\end{array}$ & $34,91 \pm 2,13 *$ & $43,73 \pm 0,47 * *$ & $36,31 \pm 1,91^{*}$ & $49,51 \pm 0,38 * *$ \\
\hline $\begin{array}{l}\text { Мышечное } \\
\text { напряжение } \\
\text { детрузора }\end{array}$ & $197,32 \pm 20,42 *$ & $254,38 \pm 19,14^{*}$ & $216,99 \pm 20,42^{*}$ & $258,61 \pm 17,60^{* *}$ \\
\hline
\end{tabular}

Прим.: ${ }^{*}-\mathrm{p}<0,05$ - уровень значимости; ${ }^{* *} \mathrm{p}<0,01$ - уровень значимости 


\section{Вывод:}

1. Принципиальное преимущество использования математического моделирования в медико-организационных исследованиях состоит в том, что наличие адекватных моделей помогает избежать неэффективных мероприятий, снизить риск экономических потерь при внедрении новой тактики медицинского обслуживания всех поступающих в больницу контингентов больных.

2. При лечении клапана уретры новым хирургическим методом коэффициент уретрального сопротивления уменьшается, живое сечение уретры увеличивается при мочеиспускании при удалении клапана металлическим вальватомом по сравнению с удалением клапана эндоскопическим путем.

3. Скорость мочеиспускания увеличивается и внутрипузырное давление мочеиспускания после удаления клапана уретры металлическим вальватомом нормализуется больше в сравнении с удалением клапана эндоскопическим путем.

4. На основании полученных данных можно сделать вывод, что благодаря круговому иссечению клапана уретры в раннем, и в отдаленном периоде после операции по физиологическим параметрам акта мочеиспускания лучшие результаты наблюдались при применении металлического вальватома. Эти преимущества нового метода приводят быстрому восстановлению нормальной уродинамики в верхних и нижних мочевыводящих путей.

Таким образом, на основании вышеизложенных математических моделей, можно отметить, что при оперативном лечении клапана уретры у детей более оптимальным способом лечения является удаление клапана уретры металлическим вальватомом.

\section{ЛИТЕРАТУРА}

1. Абдушукуров А.А Теории вероятности и математическая статистика. - Ташкент. 2010. - 169 c.

2. Арипов М. Прикладная математика в естествознании и технологии. - Т. 2012. $562 \mathrm{c}$.

3. Гельдт В.Г., Кузовлева Г.И. Диагностика пороков мочевыделительной системы у новорожденных и грудных детей. // Педиатрия. 2006. -№1. -C.87-94.

4. Байбарина Е.Н., Дегтяров Д.Н., Кучеров Ю.И., и соавт., Совершенствование ранней хирургической помощи детям с врождеными пороками развития. // Росс. вестн. перинатал. и педиатрии. - М., 2011. - №2. - С.12-19.

5. Pauline M. L. Hennus, Geert J. M. G. van der Heijden, J. L. H. Ruud Bosch, Tom P. V. M. de Jong, and Laetitia M. O. de Kort1. A Systematic Review on Renal and Bladder Dysfunction after Endoscopic Treatment of Infravesical Obstruction in Boys. PLoS One. 2012; 7(9): e44663.

6. Renkema KY, Winyard PJ, Skovorodkin IN, Levtchenko E, Hindryckx A. EUCAKUT consortium. Novel perspectives for investigating congenital anomalies of the kidney and urinary tract (CAKUT). Nephrol Dial Transp. 2011 Dec;26(12):3843-51.

7. Sinha A, Bagga A, Krishna A, Bajpai M, Srinivas M, Uppal R, Agarwal I. Revised guidelines on management of antenatal hydronephrosis. Indian J Nephrol. 2013 Mar; 23(2): 83-97/

8. Kari JA, El-Desoky S, Farag Y, Mosli H, Altyieb AM, Al Sayad A, Radawi O, Ghabra H, Basnawi F, Bahrawi O, Singh A, Farsi H. Renal impairment in children with posterior urethral valves. Ped. Nephrol. Jun;28(6):927-31.

9. Rasouly HM, Lu W. Lower urinary tract development and disease. Wiley Interdiscip Rev Syst Biol Med. 2013 May-Jun; 5(3):307-42.

10. Ruano R. Fetal surgery for severe lower urinary tract obstruction. Prenat Diagn. 2011 Jul;31(7):667-74 
11. Odubanjo MO, Oluwasola AO, Kadiri S. The epidemiology of end-stage renal disease in Nigeria: the way forward. Int Urol Nephrol. 2011 Sep;43(3):785-92.

12. Okafor HU, Ekenze SO, Uwaezuoke SN. Posterior urethral valves: determinants of outcome in a developing country. J Paediatr Child Health. 2013 Feb;49(2):115-9.

13. Suleiman SM. Primary ablation of posterior urethral valves in low-birth-weight infants by visually driven Fogarty embolectomy catheters. J Urol. May 2009, 181 (5): 2284-9

14. [Sudarsanan B, Nasir AA, Puzhankara R, Kedari PM, Unnithan GR, Damisetti KR. Posterior urethral valves: a single center experience over 7 years. Pediatr Surg Int. 2009 Mar; 25(3):283-7.

15. Manzoni C., Valentini A.L. Posterior urethral valves // Rays. -2002. -Vol.27, -№2 P.131-134.

16. Ban Hani O., Prelog K., Smith G.H. A method to assess posterior urethral valve ablation // J. Urol. - 2006. - Vol.176, №1. - P. 303-305.

17. Geavlete P., Cauni V., Georgescu D. Value of preoperative urethral ultrasound in optic internal urethrotomy // Eur. Urol. -2005. -Vol.47, -№6. -P.865-871.

18. Smeulders N, Makin E, Desai D. The predictive value of a repeat micturating cystourethrogram for remnant leaflets after primary endoscopic ablation of posterior urethral valves. J Pediatr Urol. 2011 Apr; 7(2):203-8.

19. Боровиков В.П., Боровиков И.П. Statistika: Статистический анализ и обработка данных в среде Windows.// - М. ИИД Филин. 1998., 608 с.

20. Банержи А. Медицинская статистика понятным языком: вводный курс/ пер. с анг. Под ред. В.П. Леонова. // - М.: Практическая медицина, 2007. $288 \mathrm{c}$.

21. Воропаева О.Ф., Шокин Ю.И. Вычислительные методы в медицине. - М, Вычислительные технологии. 2012. - Т. 17. - № 4. - С. 29-55.

22. Ивченко Г.И., Медведев Ю.И. Введение в математическую статистику. - М, 2010. -600c. 